\title{
Nuclear translocation of small $G$ protein RhoA via active transportation in gastric cancer cells
}

\author{
JIN XU*, YUEYING LI*, XIAOMING YANG, YONGCHANG CHEN and MIN CHEN \\ School of Medical Science and Laboratory Medicine, Jiangsu University, Jiangsu, Zhenjiang 212013, P.R. China
}

Received May 22, 2013; Accepted July 2, 2013

DOI: $10.3892 /$ or.2013.2638

\begin{abstract}
Recent studies have shown the localization of RhoA in the cell nucleus, in addition to its cellular distribution in the cytosol and cell membrane. Our previous results that a high amount of RhoA was detected in gastric cancer cell nucleus and application of anticancer drug Taxol could reduce RhoA nuclear localization, suggest a relationship between nuclear translocation of RhoA and tumor progression. However, the mechanism and biological function of RhoA nuclear translocation remain unclear. The aim of the present study was to explore the molecular mechanism(s) for RhoA protein entering the nucleus in the human gastric cancer cell line AGS. Using immunofluorescence microscopy we observed not only a partial colocalization of RhoA with importin $\alpha$, mainly surrounding and on the nuclear membrane, but also an intensive colocalization of RhoA with $\mathrm{NF}-\kappa \mathrm{B}$ P50 in both cytoplasm and nucleus, particularly in the cell nucleoli. A strong association between RhoA and importin $\alpha$ as well as RhoA and NF- $\kappa$ B P50 was revealed by co-immunoprecipitation and western blotting. Moreover, AGS cells treated with the nuclear export inhibitor, leptomycin B (LMB), showed an increase of RhoA protein amount in the nucleus, indicating an active transport process for nuclear export of RhoA. Taken together, our results suggest that nuclear translocation of RhoA in AGS cells is via active transportation, a process through importin $\alpha$ in a NF- $\kappa \mathrm{B}$-dependent manner.
\end{abstract}

\section{Introduction}

Nuclear pore complexes (NPCs) connect the nuclear interior with the cytoplasm and control the exchange between the two compartments. They are built from nucleoporins $(1,2)$ and are equipped with a sieve-like barrier that is freely permeable for small molecules, but becomes increasingly restrictive as inert mobile species approach or exceed $5 \mathrm{~nm}$ in diameter (3).

Correspondence to: Professor Min Chen, School of Medical Science and Laboratory Medicine, Jiangsu University, $301 \mathrm{XueFu}$ Road, Jiangsu, Zhenjiang 212013, P.R. China

E-mail: chenmina88@yahoo.com

*Contributed equally

Key words: RhoA, cell nucleus, active transportation, human cancer cells
This limit corresponds to a mass of $\geq 30 \mathrm{kDa}$ for spherical proteins whose transportation needs nuclear transport receptors (NTRs). Serving as the NTRs, the importin $\alpha$ and $\beta$ target hundreds of proteins to NPCs and facilitate their translocation across the nuclear envelope. Importin $\alpha$ binds to classical nuclear localization signal (NLS)-containing proteins and links them to importin $\beta$, the karyopherin that ferries the ternary complex through the NPCs (4).

RhoA, with molecular mass of $21 \mathrm{kDa}$, is the most extensively studied member of the Rho GTPase family which belongs to the Ras super-family of small $\mathrm{G}$ proteins. It has been reported to regulate many biological activities including gene transcription (5) and tumor progression (6). RhoA activation is generally associated with invasive growth and metastasis. RhoA overexpression is found in many human cancers, likely contributing to the loss of growth control and the invasive phenotype of cancer cells, whereas RhoA inhibition decreases tumor cell invasion and metastasis.

Recent studies (7-9) have indicated that the distribution of RhoA was not only in the membrane and in the cytoplasm but also in the nucleus. Further investigations in our research group showed that the nucleus localization of RhoA was affected by many factors, such as inflammatory factors and antimicrotubule agents (10).

RhoA has a close relationship with $\mathrm{NF}-\kappa \mathrm{B}$, a focal point of many signal transduction pathways (11). NF- $\kappa \mathrm{B}$ has double distribution in the cytoplasm and nucleus in many cell lines, and highly expressed at inflammation. It is composed of protein dimers, such as the heterodimer p50/p65. Classical NLSs are found in both p50 and p65 (12). In the nucleus, RhoA

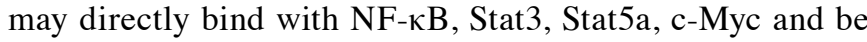
involved in regulation and control role of genetic transcription $(13,14)$. It has been reported that statins exert a positive or negative modulation on $\mathrm{NF}-\kappa \mathrm{B}$ through the involvement of RhoA, but the exact mechanism has not yet been clarified (15). Results from Chang and coworkers (16) provide evidence that Ras-induced RhoA and NF- $\kappa \mathrm{B}$ activation was involved in the invasion/migration of bladder cancer. Furthermore, LPS-induced nuclear translocation of RhoA is found to be dependent on NF- $\kappa \mathrm{B}$ in rat lung epithelial cancer cells (17). The underlying mechanism(s) for RhoA entering the nucleus is still unclear and warrants further investigation.

The biochemical characteristic of RhoA might incur the thought that RhoA enters the nucleus through simple diffusion, because it is a small molecule protein and does not 
have any classical NLS. However, based on our experiments, we questioned whether RhoA might enter the nucleus via active transportation with the help of NTRs (importin $\alpha / \beta$ ) through composing complex with other protein(s) containing NLS. Thus, the mechanism(s) on RhoA nuclear translocation requires further elucidation. In the present study, we took the $N F-\kappa B$ as the entry point to explore the manner in which RhoA enters the cell nucleus in the human gastric cancer cell line AGS.

\section{Materials and methods}

Cell lines and cell culture. Human gastric cancer cell line AGS was obtained from the Institution of Cell Biology of the Chinese Academy of Sciences (Shanghai, China). The cells were maintained in DMEM supplemented with $10 \%$ FBS at $37^{\circ} \mathrm{C}$ in a humidified atmosphere of $5 \% \mathrm{CO}_{2}$. The medium was changed every two days and the cells were kept at subconfluence.

Reagents. Dulbecco's modified Eagle's media (DMEM) and fetal bovine serum (FBS) were purchased from Gibco (Grand Island, NY, USA). Antibodies against RhoA (cat. no. sc-418) and NF-кB P50 (cat. no. sc-114) were purchased from Santa Cruz Biotechnology, Inc. (Santa Cruz, CA, USA). Monoclonal anti-importin $\alpha$ antibody (no. I1784) and nuclear fluorochrome Hoechst 33342 were purchased from Sigma (St. Louis, MO, USA). Leptomycin B (LMB) was purchased from Beyotime Institute of Biotechnology (Shanghai, China). Nuclear and Cytoplasmic Extract kit (cat. no. KC-435) and the antibody against GAPDH (glyceraldehyde phosphate dehydrogenase) were purchased from Kangcheng Bio-tech (Hangzhou, China). FITC, TRITC and horseradish peroxidase (HRP)-conjugated secondary antibodies were purchased from Jackson ImmunoResearch Laboratories (West Grove, PA, USA). Electrochemiluminescence (ECL) reagents were bought from Amersham Biosciences (Buckinghamshire, UK).

Preparation of cytoplasmic and nuclear protein samples. The cytoplasmic and nuclear proteins were extracted according to the instructions of the manufacturer of the Nuclear and Cytoplasmic Extract kit (Kangcheng Bio-tech).

Immunofluorescence microscopy. AGS cells grown on cover slips were fixed with freshly prepared paraformaldehyde (40 g/l in PBS) for $30 \mathrm{~min}$. After penetration with $30 \mathrm{ml} / 1$ Triton X-100 and blocking with $30 \mathrm{~g} / \mathrm{l}$ bovine serum albumin (BSA), the cells were incubated with the primary antibody at $4^{\circ} \mathrm{C}$ overnight $(\mathrm{o} / \mathrm{n})$ followed by an another incubation with fluorescein isothiocyanate (FITC) and/or tetrarhodamine isothiocyanate (TRITC)-conjugated second antibody for $1 \mathrm{~h}$ at room temperature (RT). Hoechst $33342(0.2 \mathrm{mM})$ was employed in the last incubation of $10 \mathrm{~min}$ to reveal the cell nuclei. AGS cells were washed three times after each incubation. The distribution of target protein of the cells was analyzed by fluorescence microscopy.

Western blotting and quantification. The sample proteins were run on 10/12.5\% SDS-polyacrylamide gels, and were transferred onto polyvinyl difluoride (PVDF) membranes (Bio-Rad
Laboratories, Hercules, CA, USA). The PVDF membranes were firstly blocked with 5\% milk in TBS-T ( $\mathrm{NaCl} 80 \mathrm{~g} / 1, \mathrm{KCl}$ $2 \mathrm{~g} / \mathrm{l}$, Tris-HCl $30 \mathrm{~g} / \mathrm{l}$, Tween-20 0.1\%, $\mathrm{pH}$ 7.4) for $1 \mathrm{~h}$ at room temperature and then incubated with the primary antibodies at $4^{\circ} \mathrm{C}$ overnight. After the incubation of membranes with the horseradish peroxidase-conjugated secondary antibodies for $1 \mathrm{~h}$ at room temperature, ECL reagents were used to show the positive bands on the membrane. The bands were detected by Typhoon 9400 (GE Healthcare, Piscataway, NJ, USA). To quantify the protein amount of RhoA, western blot results were further analyzed by SPSS 13.0 Tool software (SPSS Inc.) and the volume ratio of RhoA/GAPDH input was calculated and presented.

Co-immunoprecipitation assay. Immunoprecipitated AGS cells were lysed in TNEN buffer $(50 \mathrm{mM}$ Tris- $\mathrm{HCl}, \mathrm{pH} 7.2$, $100 \mathrm{mM} \mathrm{NaCl}, 2.5 \mathrm{mM}$ EDTA, 0.5\% NP-40). The antibodies against RhoA, importin $\alpha, \mathrm{NF}-\kappa \mathrm{B}$ and isotype matched $\mathrm{IgG}$ were used for immunoprecipitation, respectively. Immunoprecipitates were analyzed by western blotting as described above, using anti-importin $\alpha$, anti-NF- $\mathrm{B}$ or anti-RhoA antibody for corresponding protein. For analyzing proteins in cell fractions, cells were first lysed in TNEN buffer, and then extracted for nuclear and cytoplasmic proteins as described above. Proteins in cell fractions were further immunoprecipitated with an antibody against RhoA and detected by western blotting, using anti-NF- $\kappa \mathrm{B}$ antibody.

\section{Results}

The nuclear localization of RhoA in the human gastric cancer cell line AGS. Immunofluorescence microscopy showed that in the AGS cells, RhoA protein was localized on the membrane, in the cytoplasm and in the cell nucleus. Within the nucleus its predominant localization was in the nucleolus (Fig. 1A and B). Western blotting revealed similar subcellular distribution of RhoA (Fig. 1C). Quantification of protein amount (Fig. 1D) confirmed that RhoA concentrated in the nucleus of AGS cells.

Intracellular colocalization of RhoA with importin $\alpha$ and $N F-\kappa B$ P50. Importin $\alpha$ recruits cargo at low RanGTP levels in the cytoplasm, releases the cargo upon RanGTP binding into the nucleus, and returns RanGTP bound to the cytoplasm. There, GTP hydrolysis triggers dissociation of the importin-RanGTP complex and allows the importin $\alpha$ to bind a next cargo molecule (18). Thus, importin $\alpha$ has subcelluar distribution in both cytoplasm and nucleus. To determine whether importin was involved in the nulear translocation of RhoA, double-labeling immunofluorescence was performed. A partial colocalization of RhoA with importin $\alpha$ was observed, mainly surrounding and on the nuclear membrane in AGS cells (Fig. 2A, arrows). Importantly, an intensive colocalization of RhoA with NF- $\mathrm{B}$ P50 was found in both cytoplasm and nucleus, particularly in the cell nucleoli (Fig. 2B, arrows).

Co-immunoprecipitation of RhoA with importin $\alpha$ and $N F-\kappa B$ P50. To verify the findings from double labeling immunofluorescence, co-immunoprecipitation (Co-IP) assay 

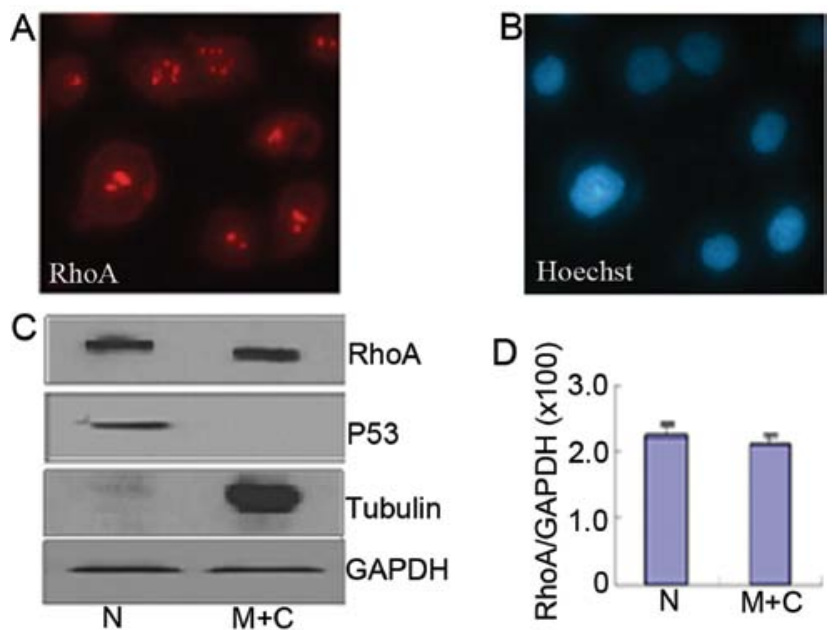

Figure 1. Subcellular localization of RhoA in human gastric cancer cell line AGS. (A) Immunofluorescence staining: RhoA was immunofluorescently stained with a specific $\mathrm{mAb}$ and the result showed its localization on the membrane, in the cytosol and in the nucleus. (B) Corresponding nuclear staining with Hoechst 33342. (C) Western blotting: localization of RhoA protein was determined in the nuclear fraction and the fraction of the membrane and cytosol of AGS cells. P53 and tubulin were employed as the markers for the nuclear and cytosolic fractions, respectively. GAPDH expression served as a loading control. (D) Volume ratio of RhoA/GAPDH input: western blotting results were analyzed by SPSS 13.0 Tool software for the calculation on the volume ratio of RhoA/GAPDH input. Each bar represents the means $\pm \mathrm{SE}$ obtained from three independent experiments. N, nucleus; $\mathrm{M}+\mathrm{C}$, membrane and cytosol.
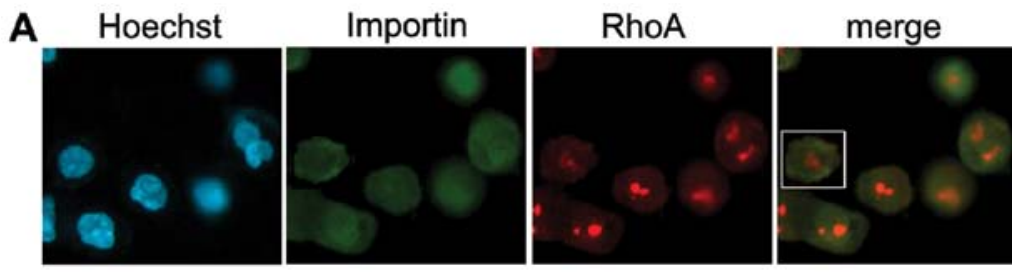

enlargement
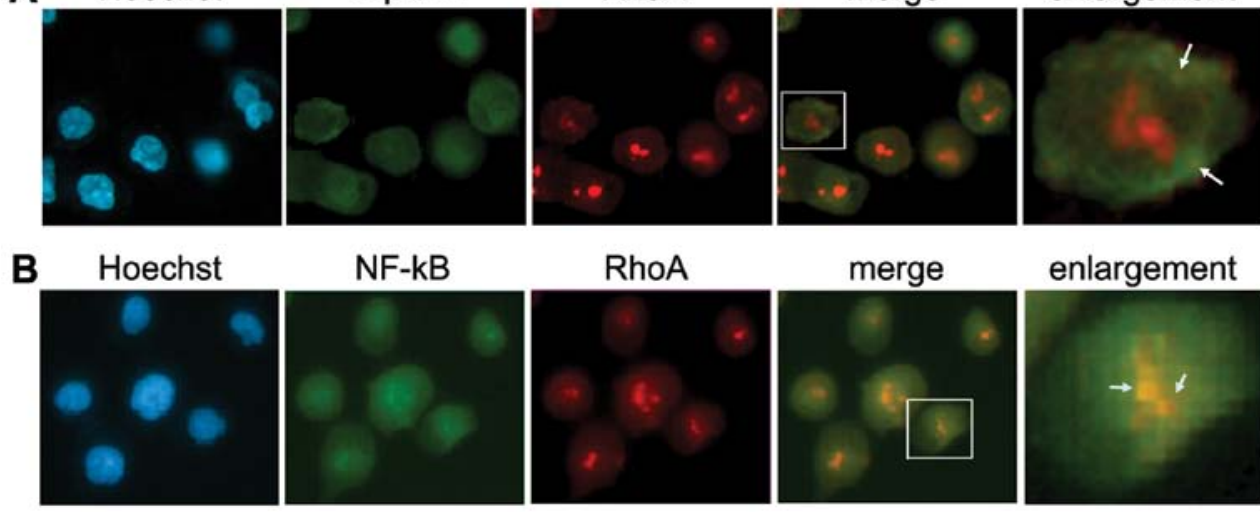

Figure 2. Subcellular colocalization of RhoA with importin and NF- $\mathrm{BB}$ in AGS cells by immunofluorescence microscopy. The cells were stained with nuclea dye Hoechst 33342 (blue), with mouse antibody against RhoA (red) and with rat antibodies against importin $\alpha$ (A) or NF-kB P50 (B) (green). Arrows in A indicate the colocalization of RhoA with importin $\alpha$ surrounding and on the nuclear membrane. Arrows in B indicate the colocalization of RhoA with NF- $\mathrm{kB}$ P50 in the nucleoli.
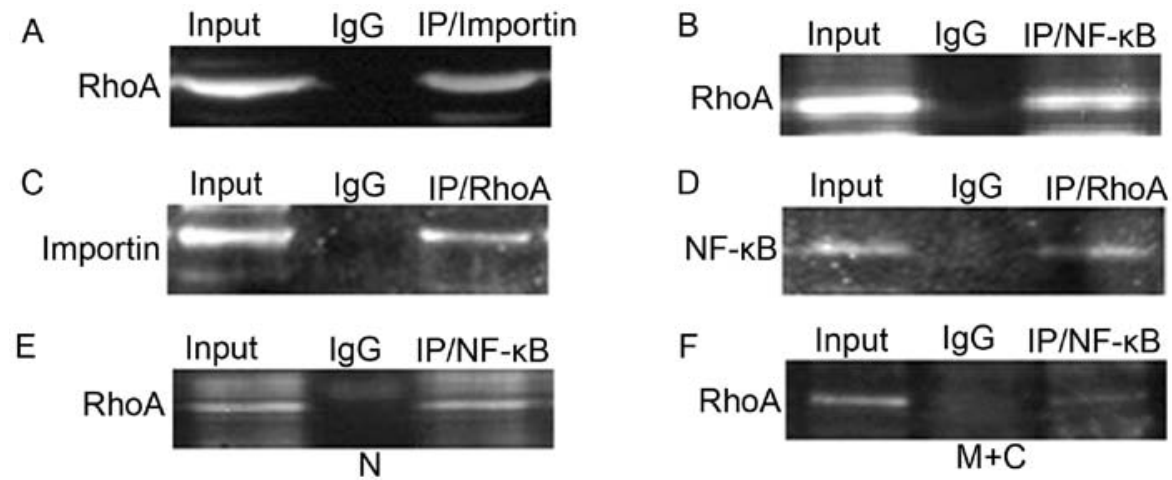

Figure 3. Co-immunoprecipitation of RhoA with importin $\alpha$ and NF- $\mathrm{kB}$ in AGS cells. The binding between RhoA and importin $\alpha / \mathrm{NF}-\kappa \mathrm{B}$ P50 were detected using the co-immunoprecipitation assay. (A) Whole cell extracts immunoprecipitated with the antibody against importin $\alpha$ and detected with RhoA antibody. (B) Whole cell extracts immunoprecipitated with the antibody against NF- $\mathrm{kB}$ P50 and detected with RhoA antibody. (C) Whole cell extracts immunoprecipitated with RhoA antibody and detected with the antibody against importin $\alpha$. (D) Whole cell extracts immunoprecipitated with RhoA antibody and detected

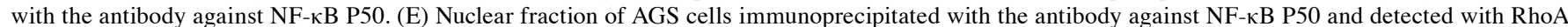
antibody. (F) Fraction of membrane and cytosol of AGS cells immunoprecipitated with the antibody against NF-kB P50 and detected with RhoA antibody. N, nucleus; $\mathrm{M}+\mathrm{C}$, membrane and cytosol. 

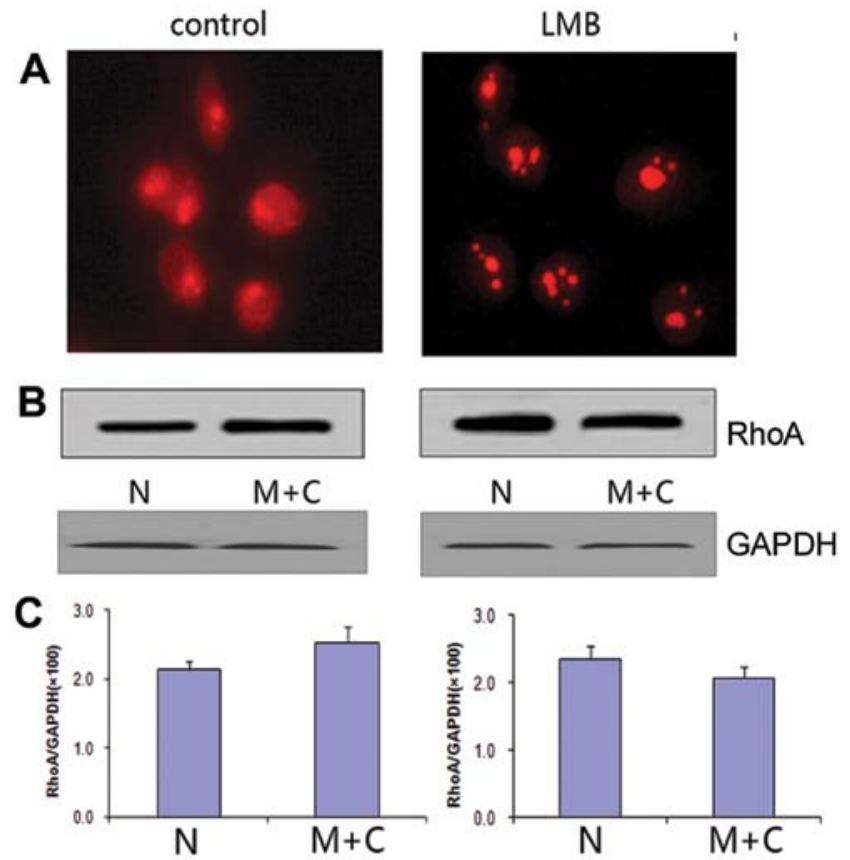

Figure 4. Subcellular distribution of RhoA in AGS cells upon nuclear export inhibition. AGS cells were left either untreated or treated with $(20 \mathrm{ng} / \mathrm{ml})$ leptomycin B (LMB) for $12 \mathrm{~h}$. (A) Indirect immunofluorescence of untreated and LMB-treated AGS cells. (B) Western blotting of untreated and LMBtreated AGS cell fractions. GAPDH served as a loading control. (C) Volume ratio of RhoA/GAPDH input: western blotting results were analyzed by SPSS 13.0 Tool software for the calculation on the volume ratio of RhoA/GAPDH input. Each bar represents means \pm SE obtained from three independent experiments. N, nucleus; $\mathrm{M}+\mathrm{C}$, membrane and cytosol.

was performed for protein interactions. Consistent with our colocalization results, there was a strong association between RhoA and importin $\alpha$ (Fig. 3A and C) as well as RhoA and NF- $\mathrm{B}$ P50 (Fig. 3B and D) in AGS cells. Further investigation on cellular fractionation showed that RhoA bound NF- $\mathrm{KB}$ P50 in the cytoplasmic and membrane fraction (Fig. 3F) and more profound in the nuclear fraction of AGS cells (Fig. 3E).

Distribution of RhoA within the nucleus upon nuclear export inhibition. To acquire further evidence for the molecular mechanism of RhoA nuclear transportation, AGS cells were treated with the nuclear export inhibitor, leptomycin B (LMB) for $12 \mathrm{~h}$. Immunofluorescence revealed a reduction of RhoA signal in the cytoplasm but a strong accumulation of RhoA in the nucleoli of LMB-treated cells (Fig. 4A). Western blot assay confirmed that the protein amount of RhoA was highly increased in the nucleus of LMB-treated AGS cells (Fig. 4B and C).

\section{Discussion}

In the classical nuclear import pathway (19), a protein containing a classical basic nuclear localization signal (NLS) is imported by a heterodimeric import receptor consisting of the $\beta$-karyopherin importin $\beta$, which mediates interactions with the nuclear pore complex, and the adaptor protein importin $\alpha$, which directly binds the classical NLS. Over the past twenty years, the universe of basic transport sequences has been greatly expanded leading to the definition of classical NLSs and, more recently, non-classical NLSs, which can be more complex in sequence, length and amino acid composition (20).

In the small $\mathrm{G}$ protein family only a small part of members has NLSs, e.g., Rac1 and R-Ras. In spite of RhoA being a small molecule protein and lacking NLSs, it is hard to conclude that nuclear translocation of RhoA is simply through diffusion, because RhoA content is higher in the nucleus than in the cytoplasm in some cancer cells $(7,10)$ and its nuclear translocation has the intimate connection with the inflammation and cancer.

Serving as a focal point of many signal transduction pathways, NF- $\kappa \mathrm{B}$ regulates the expression of genes involved in inflammation, cellular proliferation and apoptosis (21). Activation of $\mathrm{NF}-\kappa \mathrm{B}$ is followed by its rapid translocation into the nucleus where it activates the transcription of numerous genes including those encoding for cytokines and cell adhesion molecules. NF- $\kappa \mathrm{B}$ contains classical NLSs and enters the nucleus through active transportation with the help of importin $\alpha$ (12).

Consistent with the previous reports, the present research results confirmed the subcellular localization of RhoA on the membrane and in the cytoplasm and cell nucleus of human gastric cancer AGS cells. Furthermore, RhoA colocalized partially with importin $\alpha$ on and surrounding the nuclear membrane and intensively with $\mathrm{NF}-\kappa \mathrm{B}$ P50 in both cytoplasm and nucleus, particularly in the cell nucleoli. A strong association between RhoA and importin $\alpha$ as well as RhoA and NF- $\kappa$ B P50 was verified by co-immunoprecipitation and western blotting, indicating RhoA, NF- $\kappa \mathrm{B}$ and importin $\alpha$ bound together in the cytoplasm and nucleus. Since RhoA cannot be recognized and bound directly by importin $\alpha$, a possible explanation is that RhoA might form a complex with $\mathrm{NF}-\kappa \mathrm{B}$, probably also other proteins, and thereby indirectly binding to importin $\alpha$. These results suggest that the RhoA entry to the nucleus is via active transportation with the help of NTR importin $\alpha$ through composing complex with $\mathrm{NF}-\kappa \mathrm{B}$ in human gastric cancer AGS cells.

Cancer cells utilize the normal processes of nuclearcytoplasmic transport through the nuclear pore complex of a cell to effectively evade anti-neoplastic mechanisms (22). Karyopherins include both importins and exportins (23). Leptomycin B (LMB), the first identified small molecule inhibitor of CRM1 (also known as exportin-1), has greatly facilitated investigation to uncouple nuclear export from import and to analyze shuttling proteins via nucleocytoplasmic transport (24). However, no small molecule inhibitor of nuclear import has been described up to now (25). A significant increase of nuclear RhoA in AGS cells after LMB treatment implies that the molecular mechanism of RhoA protein transport out of the nucleus is also through the active transportation pathway.

The intracellular location of a protein is crucial to its functioning in a cell. As nuclear translocation of RhoA promotes the cancer progression, elucidating the nuclear functioning of RhoA will be the future task and may provide a new approach to treating cancer.

\section{Acknowledgements}

The present study was supported by the Specialized Research Fund for Senior Personnel Program of Jiangsu University 
(no. 11JDG129), the Postdoctoral Foundation of China (no. 2012M521018) and the Jiangsu province (no. 1201025B) to Y.L. and by the Zhenjiang Social Development Project (no. SH2010004) to X.Y.

\section{References}

1. Labokha AA, Gradmann S, Frey S, Hülsmann BB, Urlaub H, Baldus $M$ and Görlich D: Systematic analysis of barrier-forming FG hydrogels from Xenopus nuclear pore complexes. EMBO J 23: 204-218, 2013.

2. Hetzer MW and Wente SR: Border control at the nucleus: biogenesis and organization of the nuclear membrane and pore complexes. Dev Cell 17: 606-616, 2009.

3. Mohr D, Frey S, Fischer T, Güttler T and Görlich D: Characterisation of the passive permeability barrier of nuclear pore complexes. EMBO J 28: 2541-2553, 2009.

4. Goldfarb DS, Corbett AH, Mason DA, Harreman MT and Adam SA: Importin $\alpha$ : a multipurpose nuclear-transport receptor. Trends Cell Biol 14: 505-514, 2004.

5. Sotiropoulos A, Gineitis D, Copeland J and Treisman R: Signalregulated activation of serum response factor is mediated by changes in actin dynamics. Cell 98: 159-169, 1999.

6. Kamai T, Yamanishi T, Shirataki H, Takagi K, Asami H, Ito Y and Yoshida K: Overexpression of RhoA, Rac1, and Cdc42 GTPases is associated with progression in testicular cancer. Clin Cancer Res 10: 4799-4805, 2004.

7. Li Y, Chen Y, Tao Y, Xu J and Chen M: RhoA protein is generally distributed in the nuclei of cancer cells. Oncol Rep 24: 1005-1009, 2010.

8. Dubash AD, Guilluy C, Srougi MC, Boulter E, Burridge K and García-Mata R: The small GTPase RhoA localizes to the nucleus and is activated by Net1 and DNA damage signals. PloS One 6: e17380, 2011

9. Guilluy C, Dubash AD and García-Mata R: Analysis of RhoA and Rho GEF activity in whole cells and the cell nucleus. Nat Protoc 6: 2050-2060, 2011.

10. Li Y, Chen Y and Xu J: Factors influencing RhoA protein distribution in the nucleus. Mol Med Rep 4: 1115-1119, 2011.

11. Okamoto T, Sanda T and Asamitsu K: NF- $\mathrm{B}$ signaling and carcinogenesis. Curr Pharm Des 13: 447-462, 2007.

12. Fagerlund R, Kinnunen L, Köhler M, Julkunen I and Melén K $\mathrm{NF}-\{$ kappa\}B is transported into the nucleus by importin \{alpha\} 3 and importin \{alpha\}4. J Biol Chem 280: 15942-15951, 2005.
13. Tonozuka Y, Minoshima Y, Bao YC, et al: A GTPase-activating protein binds STAT3 and is required for IL-6-induced STAT3 activation and for differentiation of a leukemic cell line. Blood 104: 3550-3557, 2004

14. Benitah SA, Valerón PF, van Aelst L, Marshall CJ and Lacal JC: Rho GTPases in human cancer: an unresolved link to upstream and downstream transcriptional regulation. Biochim Biophys Acta 1705: 121-132, 2004.

15. Riganti C, Doublier S, Costamagna C, Aldieri E, Pescarmona G, Ghigo D and Bosia A: Activation of nuclear factor-kappa B pathway by simvastatin and RhoA silencing increases doxorubicin cytotoxicity in human colon cancer HT29 cells. Mol Pharmacol 74: 476-484, 2008.

16. Chang HR, Huang HP, Kao YL, et al: The suppressive effect of Rho kinase inhibitor, Y-27632, on oncogenic Ras/RhoA induced invasion/migration of human bladder cancer TSGH cells. Chem Biol Interact 183: 172-180, 2010.

17. Tao Y, Chen YC, Lan T, Qian H, Wang Y and Jiang L: LPS-induced nuclear translocation of RhoA is dependent on NF- $\kappa \mathrm{B}$ in the human lung cancer cell line A549. Oncol Lett 3: 1283-1287, 2012.

18. Izaurralde E, Kutay U, von Kobbe C, Mattaj IW and Görlich D: The asymmetric distribution of the constituents of the Ran system is essential for transport into and out of the nucleus. EMBO J 16 6535-6547, 1997.

19. Lange A, Mills RE, Lange CJ, Stewart M, Devine SE and Corbett AH: Classical nuclear localization signals: definition, function, and interaction with importin alpha. J Biol Chem 282: 5101-5105, 2007

20. Sessler RJ and Noy N: A ligand-activated nuclear localization signal in cellular retinoic acid binding protein-II. Mol Cell 18: 343-353, 2005.

21. Greten FR and Karin M: The IKK/NF- $\mathrm{B}$ activation pathway - a target for prevention and treatment of cancer. Cancer Lett 206: 193-199, 2004

22. Turner JG, Dawson J and Sullivan DM: Nuclear export of proteins and drug resistance in cancer. Biochem Pharmacol 83: 1021-1032, 2012

23. Fried $\mathrm{H}$ and Kutay U: Nucleocytoplasmic transport: taking an inventory. Cell Mol Life Sci 60: 1659-1688, 2003.

24. Wolff B, Sanglier JJ and Wang Y: Leptomycin B is an inhibitor of nuclear export: inhibition of nucleo-cytoplasmic translocation of the human immunodeficiency virus type 1 (HIV-1) Rev protein and Rev-dependent mRNA. Chem Biol 4: 139-1347, 1997.

25. Ambrus G, Whitby LR, Singer EL, Trott O, Choi E, Olson AJ, Boger DL and Gerace L: Small molecule peptidomimetic inhibitors of importin $\alpha / \beta$ mediated nuclear transport. Bioorg Med Chem 18: 7611-7620, 2010. 